\title{
Assessment of Knowledge on Key features of Angle's Class II Division 1 Malocclusion among Dental School Under-graduates
}

\author{
Dr Deepika Kapoor \\ Lecturer, Dept of Pedodontics, College of Medical Sciences, Bharatpur, Nepal
}

Correspondence: Dr Deepika Kapoor; Email: dpkaansh@gmail.com

\section{ABSTRACT}

Introduction: Angle in 1899 propounded the classification to recognize the malocclusion patterns. Class II Division 1 is a malalignment which can be identified by the parameters mentioned.

Objective: To assess the knowledge of dental undergraduate students about Class II Division 1 malocclusion and their opinion about the treatment or clinical approach to such patients.

Materials \& Method: The study was performed using closed questionnaire on 138 fourth and final year undergraduate dental students of College of Dental Sciences, Bharatpur, Nepal. Dental casts and photographs of a patient with Class II Division 1 were shown to the respondents to depict deviated midline, midline diastema, molar relation, increased overjet and overbite.

Result: Dental students found it easy to identify increased overjet (91.99\%), increased overbite (88.78\%), presence of diastema (84.69\%) and midline deviation (77.28\%). However, 52\% students could not identify the molar relationship. When asked about the appropriate timing for treatment; $48.8 \%$ thought it should be in deciduous dentition period, $41.6 \%$ in mixed dentition, and $9.6 \%$ in permanent dentition.

Conclusion: Fourth and final year BDS students possess fair knowledge on common parameters used to determine Class II Division 1 malocclusion but they find difficulties in compiling and applying this knowledge to orthodontic treatment concept.

Keywords: Angle's Classification, Knowledge, malocclusion, orthodontic treatment

\section{INTRODUCTION}

Sir Edward Angle in 1899 classified malocclusion on the basis of intercuspation of permanent first molars. There are many parameters to classify malocclusions into further divisions. The diagnosis of Class II Division 1 malocclusion is a critical task; which is complex and includes many parameters. ' Class II Division 1 malocclusion can present with complex skeletal and dentofacial features. Best treatment protocol can be formulated by considering maxillo-mandibular features, vertical and sagittal components of skeletal growth along with the dentofacial abnormalities. Other factors like age play an indispensible role in determining the best therapeutic plan. The timing on commencement of the treatment is very crucial. Eruption pattern of the teeth and tooth buds can be assessed to determine the dental age of the patient. ${ }^{2}$ Dental age and chronological age are crucial factors in treatment plan of a Class II Division 1 malocclusion. Another consideration for treatment planning is the pubertal growth spurt which occurs between 9-12 years in girls and $11-13$ years in boys. ${ }^{3}$
Treatment of Class II malocclusion can be rendered at three stages: pre-pubertal, pubertal and post-pubertal stages. Thus, it becomes imperative to understand these stages and diagnose the malocclusion with the decision to commence the treatment on time. The knowledge on need of the treatment and correct timing of commencement is imperative for treating the case or to refer to the specialist. ${ }^{4}$

Some patients require early treatment to modify the growth problems by functional orthopedics while others need late intervention to treat with camouflage. Some authors believe that functional appliances modify the underlying malocclusion by changing the condyle position. Such issues need to be addressed in detail with evidence about the mandibular incremental growth and changes to condyle. ${ }^{4}$

Treatment protocol for Class II malocclusion is case specific and many factors need a thorough consideration to meet the treatment goal. Many authors believe two types of protocols for the treatment of Class II malocclusion. Proponents of the 
first approach believe the treatment should be started early in the pre-adolescent period when the skeletal and molar correction can be done and the second phase of treatment include fixed orthodontics at a later stage. ${ }^{5}$ While the advocates of second protocol believe that; Class II Division 1 malocclusion should be started after the growth has ceased and rendered in a single phase where both skeletal and dental problems are addressed. ${ }^{6}$

The present study was done with an objective of assessing the knowledge of dental undergraduate students about Class II Division 1 malocclusion and their opinion about the treatment approach. During these phases, these students are often the first observant of the patients when they take clinical history and aware the patients about the treatment. Thus, assessing this knowledge will help in reviewing the dental education system and assess their role for a good referral system.

\section{MATERIALS AND METHOD}

The study was initiated with the approval from IRC in February 2018. The sample consisted of 138 fourth and final year BDS students of College of Medical Sciences, Bharatpur, Nepal. A questionnaire was used amongst the students to collect data regarding their knowledge. A closed seminar was organized to present a case report using facial photographs and dental casts of a known Class II Division 1 case. The age and gender of the patient was revealed. Those photographs and dental casts had clear depiction of clinical features including: increased overjet and overbite, spacing in maxillary anteriors, and deviated midline in mixed dentition. Cephalometric and radiographic records were not provided to simulate a normal clinical situation.

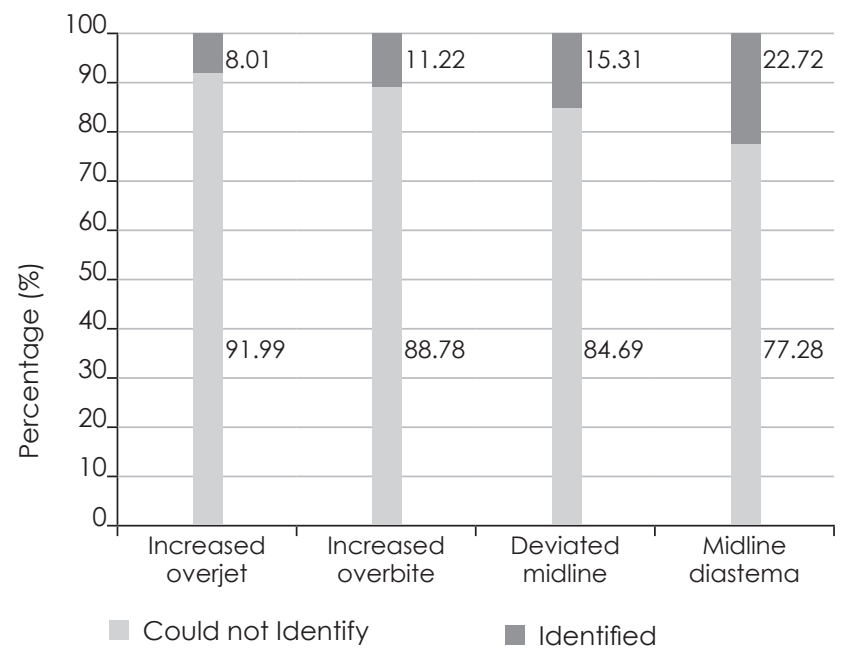

Figure 1. Identification of parameters of Class II Div 1
The students were then asked to analyze the dental casts and photographs and answer the questions regarding identification of Angle's classification of malocclusion, clinical features to base their diagnosis, treatment protocol, and referral time. All responses and student's identity were maintained confidential. The collected data were processed and analyzed using SPPS software.

\section{RESULT}

Figure 1 depicts the knowledge of the undergraduate dental students about the features of Class II Division 1 malocclusion including increased overjet and overbite, midline diastema, midline shift in the presented case. They exhibited a fair knowledge of these clinical features. Increased overjet was identified by $91.99 \%$, increased overbite by $88.78 \%$, deviated midline by $84.69 \%$ and midline diastema by $77.28 \%$.

This sample had a poor knowledge on Angle's classification and could not identify bilateral Class II molar relation (52\%). Some of them identified Class II molar relation unilaterally $(30 \%)$ and very few identified them bilaterally (18\%).

When the undergraduates were asked about the need of treatment to such case, almost all of them agreed to the need for treatment from the specialist and required referral $(97.8 \%)$ while $2.2 \%$ said; there is no need for the treatment.

Answers regarding the age of commencement for treatment showed a wide variation amongst undergraduate students. $48.8 \%$ thought it should be treated in deciduous dentition period, $41.6 \%$ in mixed dentition, while $9.6 \%$ thought, it should be commenced in permanent dentition period (Figure 2).

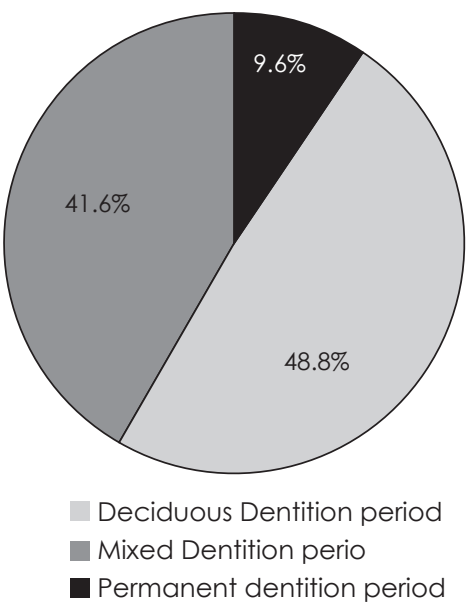

Figure 2. Opinion on timing of treatment 


\section{DISCUSSION}

The undergraduate dental curriculum has a wide coverage of orthodontic diagnosis; so that the graduates can identify the orthodontic problems and refer to the specialist. ' The curriculum should make a foundation of the knowledge required to diagnose the malocclusion correctly. ${ }^{3}$

A unique study was done in Ireland regarding undergraduate orthodontic training and their application in clinical practice. The results showed $54 \%$ positive response on academic knowledge, $60 \%$ can handle orthodontic emergencies, and $70 \%$ are aspired to go for higher education in this field. ${ }^{8}$

Many studies have assessed the knowledge of undergraduates about the concept of malocclusion, its diagnosis and treatment timing; however there have been a deficiency in knowledge. ${ }^{9}$ A similar study done on the knowledge of Angle Class I and Class III malocclusions showed similar results as our study. ${ }^{10}$ Another study done on the knowledge of Angle's Class II malocclusion in Brazil also showed similar results."

In a study done in India, the knowledge and attitude about the general principles and practices of orthodontics were assessed among the general dental practitioners and other dental specialties. The result depicted highly significant difference of knowledge amongst general dental practitioners and the specialists. ${ }^{12}$ Some studies revealed lack of knowledge about oral health among clinical students. ${ }^{13}$

After analyzing the results, we suggest more comprehensive studies to be done in this regard. Also, the curriculum should emphasize on the diagnosis of basic orthodontic problems. The basic concept is of paramount importance as against the knowledge of fabrication of orthodontic appliances. ${ }^{14}$

\section{CONCLUSION}

Dental undergraduate students of fourth and final year have a fair knowledge of common parameters used to determine Class II Division 1 malocclusion; however they find difficulties in compiling and applying this knowledge to the clinical concept. They are also uncertain about the timing of the commencement of treatment.

\section{REFERENCES}

1. Proffit WR, Fields H. Contemporary orthodontics. 3rd ed. St. Louis: Mosby; 2000.

2. O'Reilly MT, Yanniello GJ. Mandibular growth changes and maturation of cervical vertebrae - A longitudinal cephalometric study. Angle Orthod. 1988;58(2):179-84

3. Jacobs RM. Ten-year study of strategies for teaching clinical inference in predoctoral orthodontic education. J Dent Educ. 1979;41:477-8.

4. Turpin DL. The long-awaited Cochrane review of 2-phase treatment. Am J Orthod Dentofacial Orthop. 2007;132(4):423-4.

5. King GJ, MCGorray SP, Wheeler TT, Dolce C, Taylor M. Comparison of peer assessment ratings (PAR) from 1-phase and 2-phase treatments protocols of Class II malocclusion. Am J Orthod Dentofacial Orthop. 2003;123(5):489-96.

6. Keeling SD, Wheeler TT, King GJ, Garvan CW, Cohen DA, Cabassa S. Anteroposterior skeletal and dental changes after early Class II treatment with bionators and headgear. Am J Orthod Dentofacial Orthop. 1998;113(1):40-50.

7. Koroluk LD, Tulloch JF, Phillips C. Incisor trauma and early treatment for Class II Division 1 malocclusion. Am J Orthod Dentofacial Orthop. 2003;123(2):117-25

8. Fleming PS, Dowling PA. A survey of undergraduate orthodontic training and orthodontic practices by general dental practitioners. J Ir Dent Assoc. 2005;51 (2):68-72.

9. Ghafari J. Timing the early treatment of Class II, division 1 malocclusion- Clinical and research considerations. Clin Orthod Res. 1998; $1(2): 118-29$

10. Miguel JAM, Brunharo IP, Esperão PTG. Oclusão normal na dentadura mista: reconhecimento das características oclusais por alunos de graduação. Rev Dental Press Ortod Ortop Facial. 2005;10(1):59-66.

11. Canavarro C. Assessment of the orthodontic knowledge demonstrated by dental school undergraduates: recognizing the key features of Angle Class II, Division 1 malocclusion. Dental Press J Orthod. 2012;17(1):52.1-10.

12. Shastri RM, Tampure VS, Palagi FB, Shinde Sk, Ladhe K, Polepalle T. Study of the Knowledge and Attitude about Principles and Practices of Orthodontic Treatment among General Dental Practitioners and Non-orthodontic Specialties. J Int Oral Health. 2015; 7(3):44-8.

13. Ahamad S, Moyin S, Punathill S, Pawar G. Evaluation of the Oral Health Knowledge, Attitude and Behavior of the Preclinical and Clinical Dental Students. J Int Oral Health. 2015;7(6):65-70.

14. Moyers RE, Riolo ML, Guire KE, Wainright RL, Bookstein FL. Differential diagnosis in Class II malocclusions. Am J Orthod. 1980;78(5):477-94. 